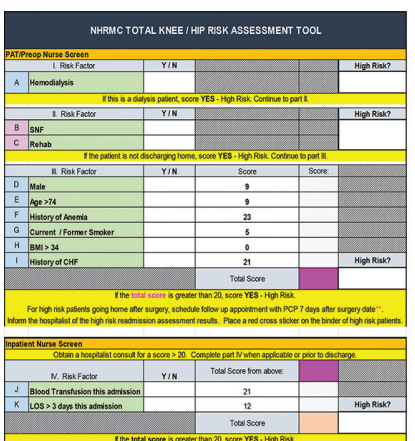

\begin{tabular}{|c|c|c|}
\hline Root Causeffator & PValue & $\begin{array}{l}\text { Segificantornot } \\
\text { gors Confidencel interal }\end{array}$ \\
\hline Gender & .004 & Yes Nales $1.5 \mathrm{X}$ more likely. \\
\hline Surgean & .462 & No \\
\hline Age 74 lears & .000 & Yes $1.8 X$ more likely \\
\hline Race & .387 & No \\
\hline Discharge Dispo:SNFF & .000 & 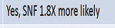 \\
\hline Blood Trenstusion & .000 & Yes, 37.7 more il ilely \\
\hline Los $>3$ days & .000 & res Los 302.25 more likely. \\
\hline $\mathrm{CHF}$ & .000 & Yes, $3.8 \mathrm{X}$ more likely \\
\hline Obesity(3MM / 35) & .308 & No \\
\hline Aremia & .000 & Yes Anemic 3 X morel lively \\
\hline Disbetic & .011 & Yes $1.5 \mathrm{~m}$ more likey \\
\hline Strole & .014 & Yes, 2 Xmore liliely \\
\hline Renalfalure & .000 & Yes 2 m more liely \\
\hline Tobaccouse & .127 & No. Consider data reliability \\
\hline
\end{tabular}

Abstract 869 Figure 2 NHRMC total knee/hip risk assessment tool

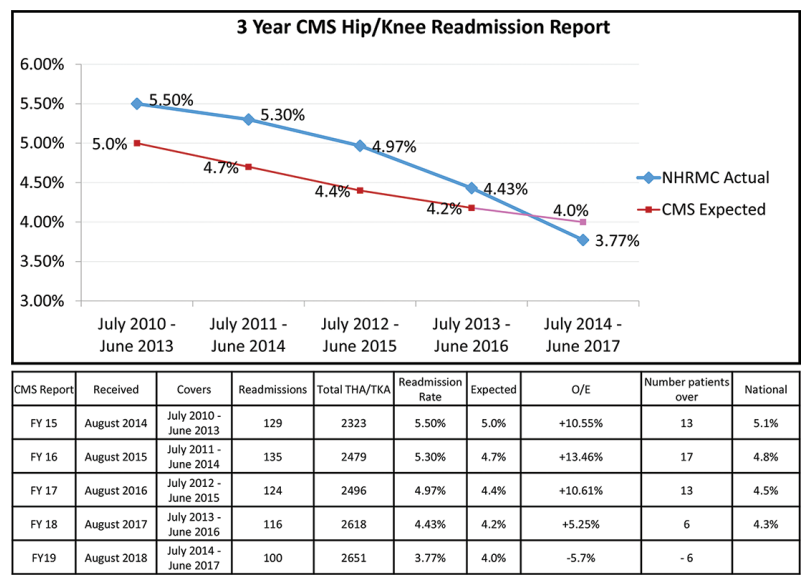

Abstract 869 Figure 33 year CMS hip/knee readmission report

more contact post discharge via phone calls focused on identified risk factors and common readmission reasons.

Results Initial CMS 3 year aggregate readmission rate was $5.5 \%$ versus $4.43 \% \mathrm{p}=0.04$ FY18 CMS report and $3.77 \%$ $\mathrm{p}=0.002$ FY'19 projected. Risk factor analysis and mirrored intervention strategies by other readmission populations and increased desire and utilisation of orthopaedic preadmission testing optimisation and anaemia clinic by other surgical populations.
Conclusions New Hanover Regional Medical Centre has statistically reduced readmissions and should receive no further Hip/Knee Arthroplasty Readmission penalties. Six sigma process of patient level analysis, statistically validated risk scoring, patient optimisation, reduced clinical variation, and risk based interventions can be utilised anywhere, with any population, to prevent unwanted outcomes.

\section{CONTINUOUS INFUSION MEDICATION SAFETY IN A PAEDIATRIC INTENSIVE CARE UNIT}

Ashley Ried. Monroe Carell Jr. Children's Hospital at Vanderbilt, US

\subsection{6/bmjoq-2017-IHI.6}

Background Failure to reliably use Computerised Physician Order Entry systems for continuous medications increases a patient's risk of experiencing adverse drug events, is outside the scope of practice for nurses and contradicts regulatory standards.

Objectives Increase the percentage of continuous medications infusing in the Paediatric Intensive Care Unit matching the current order from $74 \%$ to $90 \%$ by May 31, 2017 to eliminate adverse drug events.

Methods An interdisciplinary team conducted a Failure Modes Effects Analysis to identify failure modes and interventions. A Key Driver Diagram was then created to frame the improvement work. The first Plan-Do-Study-Act cycle ensured ordering providers read back orders on rounds to provide real-time loop closure. A Pareto chart of failures identified vasoactive medications as the largest non-compliant category, leading to another PDSA implementing vasoactive titration order-sets. Rapid cycle testing and feedback allowed the team to address concerns before full implementation.

Results Statistical process control was used for the percentage of continuous medications and vasopressors matching the provider order displayed in separate p-charts. Established rules were used for differentiating special versus common cause variation. 431 baseline observational audits revealed a mean compliance of $74 \%$ that increased to $93 \%$ after 12 months. Subset analysis of vasoactive medications showed an improvement from $38 \%$ to $89 \%$ compliance.

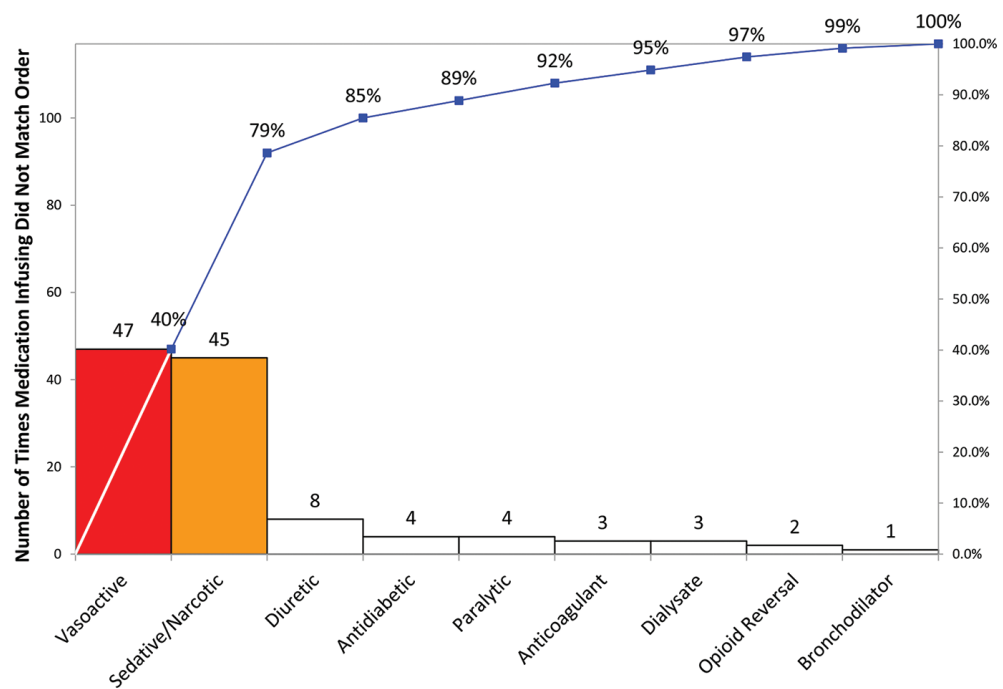

Abstract 873 Figure 1 Pareto: PICU continuous IV medication infusions by class that do not match order 6/5/16-9/27/16 


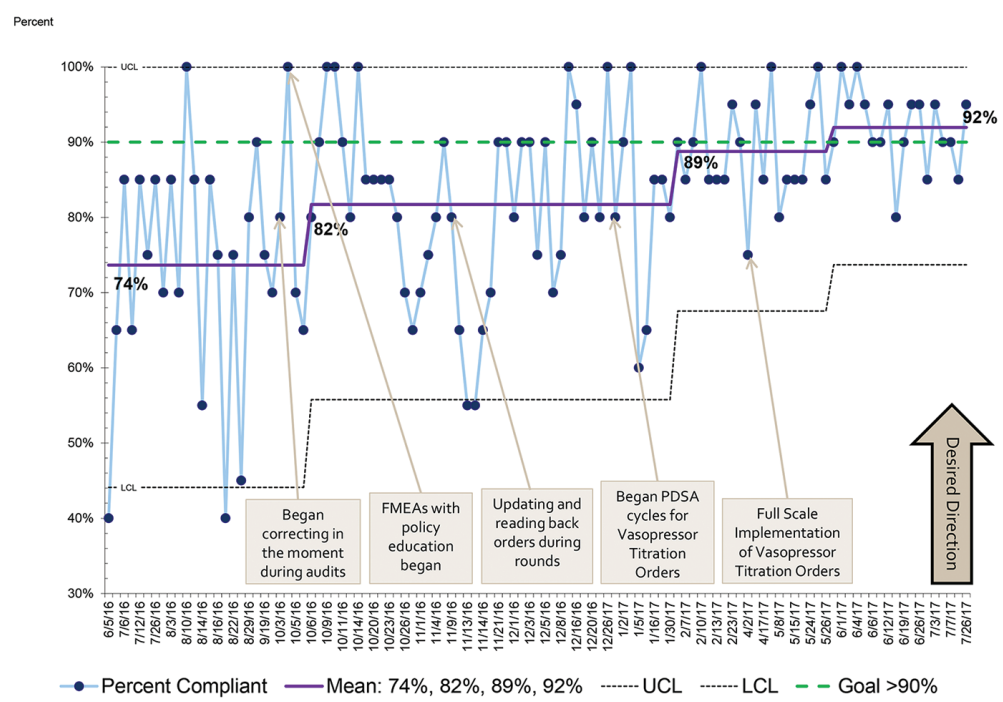

Abstract 873 Figure 2 P chart: PICU continuous infusions matching the current order group of 20

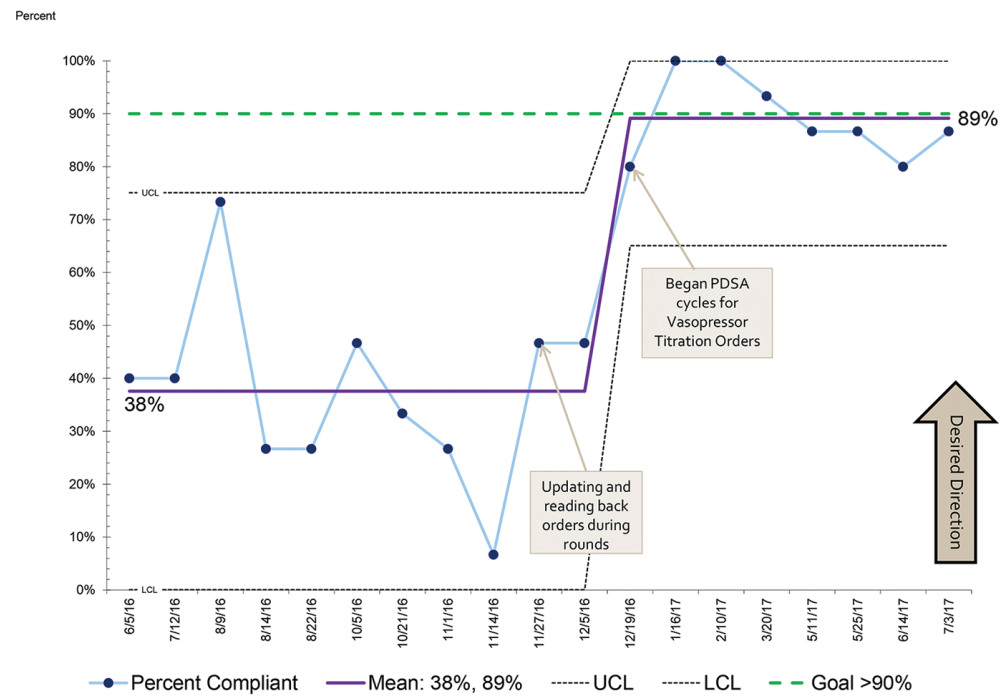

Abstract 873 Figure 3 P chart: PICU vasoactive medications matching the provider order group of 15

Conclusions Ensuring continuous medications have matching provider orders uses proven safety mechanisms, ensures nurses provide care within their scope of practice, and reduces adverse drug events. This project will be spread to the Cardiac ICU and has been used to develop guidelines for medication orders in EPIC.

\section{A QUALITY IMPROVEMENT PROGRAM TO ACHIEVE INDEPENDENCE IN HOME CENTRAL LINE CARE BY PAEDIATRIC ONCOLOGY FAMILIES}

${ }^{1}$ Chris Wong, ${ }^{2}$ Constance Barysauskas, ${ }^{3}$ Marie Desrochers, ${ }^{3}$ Margaret Brill-Conway, ${ }^{3}$ Riley Mahan, ${ }^{3}$ Kelly Eng, ${ }^{3}$ Amy Billett. ${ }^{1}$ Dana-Farber/Boston Children's Cancer and Blood Disorders Centre, US; ${ }^{2}$ Dana-Farber Cancer Institute, US; ${ }^{3}$ Blood Disorders Centre, US

\subsection{6/bmjoq-2017-IHI.7}

Background Central line associated blood stream infections (CLABSI) occur frequently in the paediatric oncology (PO) population increasing morbidity, mortality, and costs. Families in the home provide a large portion of PO external central line (CL) care with limited opportunities to develop skills that maximise best practice bundle adherence, key to infection prevention.

Objectives To achieve $>90 \%$ of families with documented independence in CL flushing by 12 months.

Methods Plan-do-study-act cycles were used to test changes from April 2016-June 2017 after identifying drivers in a pilot (June 2015-March 2016) to develop a family-centred learning curriculum. This involved return-demonstration [teach-backs (TBs)] of CL skills in front of a nurse coach during routine clinic visits and use of CL care cognitive aids. Key interventions: culture change-new expectation of $\mathrm{TB}$ participation until independent; embedding TBs into routine care with a dedicated nurse champion; developing checklists to standardise content, approach, performance, and proficiency evaluation with documentation. Statistical process control charts tracked changes over time.

Results During the pilot, $12.9 \%(22 / 171)$ participated in the TB program and $4.7 \%(n=8)$ had documented independence. After April 2016, special cause variation shifted the centre line of families' independent from $6 \%$ to $89 \%$. Participation 